\title{
THEORY OF ACCRETION DISK CORONAE
}

\author{
T. R. Kallman \\ NASA/Goddärd Space Flight Center, Greenbelt, MD USA
}

\begin{abstract}
Accretion disk coronae are likely to be the dominant site for Xray absorption and reprocessed emission in low mass X-ray binaries, and may be present in other classes of compact X-ray sources such as active galactic nuclei and cataclysmic variables. In spite of this fact, and in spite of the observational evidence for their existence, there remain many uncertainties about the structure of accretion disk coronae. This paper will discuss the coronal structure and dynamics, their Xray spectral signatures including coupling to the variability behavior of compact $\mathrm{X}$-ray sources, and the major unsolved theoretical issues surrounding them.
\end{abstract}

\section{INTRODUCTION}

Accretion disks are a common occurrence in a variety of astronomical systems, ranging from cataclysmic variables and X-ray binaries to the nuclei of active galaxies. It is likely that the disks that form as the result of accretion share many common features and differ primarily in their scale size. In many situations a large fraction of the energy of the accreted material is released as X-rays near the compact object at the disk center. The illumination of the disk surface by these $\mathrm{X}$-rays produces an accretion disk corona or wind (hereafter collectively reffered to as an ADC) which is the dominant site for the formation of spectral features observable by high resolution X-ray spectrometers.

The idea of accretion disk coronae dates back to early work on accretion disk structure by Shakura and Sunyaev (1973). The most detailed discussions of ADC structure, and those which form the basis for much of the present discussion, are those by Begelman, McKee and Shields (1982) and Begelman and McKee (1982; hereafter referred to collectively as BM), and by White and Holt (1981) and by McClintock, et al. (1982), and others referred to by these authors. Since the theory of accretion disk coronae and their implications for observations have already been discussed in detail by these authors, in this review I will concentrate on the confrontation between theory and the past and future observations of ADC sources using high resolution X-ray spectrometers. In order to do so, it is necessary to summarize some of the past theoretical results; this is done in Section II. Models for ADC X-ray spectra and the comparison with observations are described in Section III, and the prospects for future progress are discussed in Section IV. 


\section{ACCRETION DISK CORONA BASICS}

For the purpose of estimating the properties of accretion disk coronae I will assume that the accretion disk is geometrically "thin" (i.e. vertical thickness much less than radial distance from the X-ray source) and optically thick (to the cooling radiation emitted near the midplane) (see, e.g. Shakura and Sunyaev 1973; the interior structure of the disk is not of crucial importance to the corona structure), that radiation pressure and disk self-gravity are negligilble. I will also assume that the heating of the disk atmosphere is dominated by X-rays from a compact object at the disk center, that sources of mechanical heating such as convection or MHD waves are unimportant, and that the timescales for variability of the X-ray source intensity or the disk structure are long compared to the timescales affecting the corona structure.

The temperature of X-ray heated gas depends only on the shape of the $X$-ray spectrum and on the ionization parameter, $\Xi$. This quantity is defined as the ratio of the X-ray flux, F (in energy units over the band 1 - $1000 \mathrm{Ry}$ ), to the gas pressure $\mathrm{P}$, according to $\Xi=F / P c$ (c.f. Krolik, McKee, and Tarter, $1981)$. For ionization parameter values greater than a critical value, $\Xi_{c}^{*}$, the gas temperature is determined primarily by the effects of Compton scattering. The "Compton temperature" is $T_{I C}=\langle\varepsilon\rangle / 4 k$, where $\langle\dot{\varepsilon}\rangle$ is the mean photon energy. For X-ray spectra similar to those observed from LMXRB, the Compton temperature is $T_{I C} \sim 10^{7} \mathrm{~K}$. For ionization parameters less than a critical value $\Xi_{h}^{*}$, the gas temperature is determined by the effects of atomic processes; the temperature in this case is $T_{\text {atomic }} \sim 10^{4} \mathrm{~K}$. These critical ionization parameters have values $\Xi_{h} \simeq 0.3\left(T_{I C} / 10^{8} K\right)^{-3 / 2}$ and $\Xi_{c}^{*} \simeq 10$ (although various effects such as conduction, non-equilibrium effects, strong Compton cooling by the UV radiation from the disk and other sources of heating may modify these estimates). For intermediate ionization parameters, $\Xi_{h}^{*}<\Xi<\Xi_{c}^{*}$, there exists a third equilibrium temperature which is unstable according to Field's (1965) criterion. This simple $\Xi$ scaling behavior applies provided the gas is optically thin to the X-rays; the validity of this assumption will be discussed later.

The existence of an X-ray heated corona depends on the decrease of gravity with distance from the accretion disk midplane. The conditions necessary for the formation of a corona may be expressed in terms of the hydrostatic condition:

$$
\frac{d P}{d z}=-\frac{G M m_{H}}{R^{3} k T} P z=-\frac{P z}{z_{s}^{2}} \leq 0
$$

where $P$ is the gas pressure, $z$ is height above the disk midplane, $M$ is the mass of the central X-ray source, $R$ is the distance from the X-ray source and $m_{H}$ is the mean mass per particle in the gas. The scale height is $z_{s}=\sqrt{R^{3} k T /\left(G M m_{H}\right)} \doteq 8 \times$ 
$10^{7} \mathrm{~cm} R_{9}^{3 / 2} T_{7}^{1 / 2}\left(M / M_{\odot}\right)^{-1 / 2}$, and the temperature gradient in the disk atmosphere may be written:

$$
\frac{d T}{d z}=\frac{d P}{d z} \frac{d T}{d \Xi}\left(\frac{d \Xi}{d P}\right)_{F}
$$

Since $(d \Xi / d P)_{F}>0$, the necessary condition for a corona to form is that $d T / d \Xi<0$ which is satisfied for $\Xi_{h}^{*}<\Xi<\Xi_{c}^{*}$ in X-ray illuminated disks if $T_{I C} \geq 10^{7} \mathrm{~K}$.

Therefore, we expect a corona at $T=T_{I C}$ to exist wherever $\Xi \geq \Xi_{\hbar}^{*}$. Near the interface between the corona and the disk photosphere must exist a chromosphere and transition region in which X-ray heating is important, but is not sufficiently strong to heat the gas to temperatures comparable to $T_{I C}$. These regions must dominate the optical and UV appearance of X-ray illuminated accretion disks. The maximum density of the corona may then be expressed in terms of the incident X-ray flux $F$ according to:

$$
\begin{aligned}
n_{m \alpha x} & =\frac{F}{c \Xi_{c}^{+} k T_{I C}} \\
& =1.9 \times 10^{17} \mathrm{~cm}^{-2} R_{9}^{-2} L_{37} f T_{I C 7}^{-1}
\end{aligned}
$$

where $R_{9}$ is the radius in units of $10^{9} \mathrm{~cm}, \mathrm{~L}_{37}$ is the $\mathrm{X}$-ray source luminosity in units of $10^{37} \mathrm{ergs}^{-1}$, and $T_{I C 7}$ is the Compton temperature in units of $10^{7} \mathrm{~K}$. The effects of geometry and the transfer of $\mathrm{X}$-rays through the corona are combined in the factor $f=4 \pi R^{2} F / L . f=1$ corresponds to unattenuated $X$-rays normally incident on the disk. The total coronal column is $\sim n_{\max } z_{3}$ or

$$
\begin{aligned}
N_{1 o t} & =\frac{L f}{4 \pi \Xi_{c}^{*} c}\left(R k T_{\left.I C \mu m_{H} G M\right)^{-1 / 2} E\left(z_{b} / z_{s}\right)}\right. \\
& =1.5 \times 10^{25} \mathrm{~cm}^{-2} L_{37} f R_{g}^{-1 / 2} T_{I C 7}^{-1 / 2} E\left(z_{b} / z_{s}\right)
\end{aligned}
$$

where $z_{b}$ is the height of the base of the corona, $M$ is the mass of the compact object, $\mu m_{H}$ is the mean mass per particle in the gas and $E$ is a slowly varying function related to the error function. It is clear from this equation that the column density of the corona increases with decreasing distance from the source, and that when $R \geq$ 
$R_{\text {hick }}=10^{9} \mathrm{~cm} L_{37}^{2} f^{2} T_{I C 7}^{-1}$ the Thompson depth through the corona exceeds unity. This buildup must ultimately be limited by the fact that $\mathrm{X}$-rays will not penetrate to the base of a corona whose Thompson depth, $\tau_{T h}$, exceeds some critical value which we define as $\tau_{T h h_{\text {crit }}} \simeq 1-10$. Therefore we may treat the requirement $\tau_{T h} \leq \tau_{T h c_{\text {c } i t}}$ as a second ("optically thick") necessary condition. for coronal equilibrium. When this condition applies, the minimum ionization parameter in the corona may be shown to be

$$
\begin{aligned}
& \Xi_{\min }=\frac{L f \sigma_{T h}}{4 \tau_{T h \text { rit }} \pi c\left(R k T_{I C} \mu m_{H} G M\right)^{1 / 2}} E\left(z / z_{s}\right) \\
& =10 L_{37} f R_{9}^{-1 / 2} T_{I C 7}^{-1 / 2} \tau_{T h \text { cr id }}^{-1} \frac{M}{M_{\text {odot }}} E\left(z / z_{s}\right)
\end{aligned}
$$

where $\sigma_{T_{h}}$ is the Thompson cross section and $\mathrm{z}$ is height at which Thompson depth $\tau_{\text {Thcrit }}$ occurs $\left(E\left(z / z_{s}\right)\right.$ is of order unity for our fiducial parameter values and is a very slowly varying function of $R$ ). In what follows we assume $\tau_{T h}$ crit $_{1}=1$. Since $\Xi_{h}^{*} \simeq 0.1-1$ (c.f. Krolik, McKee, and Tarter, 1982) this suggests that in the inner region of the disk where the optically thick criterion applies the mean ionization parameter may be much greater than $\Xi_{c}^{*}$, and that it increases with decreasing distance from the source.

X-ray heating may also have dynamical implications for the corona. These are characterized in terms of the "escape temperature" of disk gas $T_{c s c}=$ $G M m_{H} /(k R)=1.6 \times 10^{9} K R_{9}^{-1}\left(M / M_{\odot}\right)$, which is the temperature of a gas whose thermal velocity equals the escape velocity from the disk. At radii $R \geq R_{I C}=$ $1.6 \times 10^{11} \mathrm{~cm} T_{I C 7}^{-1}\left(M / M_{\odot}\right)$ the corona temperature exceeds the escape temperature and the corona is not bound to the disk. In this region, referred to as the "wind region", the mass loss rate from the disk can greatly exceed the mass accretion rate. This can lead to a limit cycle behavior as the disk is depleted by the wind, the X-rays turn off when the depleted zone propogates inward to the X-ray source, and the disk reforms in the absence of X-rays (Shields, et al., 1986). The timescale characterizing this process is the viscous timescale in the disk (e.g. Begelman and McKee, 1982), $\Delta t \sim \Delta t_{v i s c o u s} \simeq 85 s \alpha^{-1}\left(h_{d} / R_{I C}\right)^{-2}\left(M / M_{\odot}\right)\left(R / R_{I C} T_{I C 8}\right)^{3 / 2}$

Another process which may affect the disk structure is cooling of the corona by Compton scattering. The flux at the surface of the disk from viscous energy generation has a temperature in the range $10^{4}--10^{5} \mathrm{~K}$ is $F_{d}=2.2 \times$ $10^{16} \mathrm{ergcm}^{-2} \mathrm{sec}^{-1} R_{9}^{-3}\left(M / M_{\odot}\right) L_{37}(\eta / 0.1)$. The radius at which this equals the flux from the X-ray source is $R_{e q}=2.7 \times 10^{7} \mathrm{~cm}^{-1} M / M_{\odot}\left(\frac{\eta}{0.1}\right)$; for $R \leq R_{e q}$ the disk will cool the corona to the disk photospheric temperature $T_{d \text { isk }}=\left(F_{d} \sigma\right)^{1 / 4}=$ $1.4 \times 10^{5} K \bar{R}_{9}^{-3 / 4}\left(M / M_{\odot}\right)^{1 / 4} L_{37}^{1 / 4}(\eta 0.1)^{1 / 4}$. In the outer region of the disk, where the 
corona is optically thin, the corona will be cooled by the $\mathrm{X}$-rays which pass through it and are absorbed and re-radiated by the disk photosphere. The temperature of these re-radiated X-rays is $T_{d i s k}=\left(F_{x} / \sigma\right)^{1 / 4}=3.4 \times 10^{5} K R_{9}^{-1 / 2} f^{1 / 4} L_{37}^{1 / 4}$. Since the flux at this temperature will at most equal that of the direct $X$-rays, this effect will reduce the corona temperature by a factor of 2 .

In the optically thin coronal region the emission measure of the corona is

$$
\begin{aligned}
E M_{t h i n} & =\left(\frac{f L}{c k T_{I C} \Xi_{c}^{*}}\right)^{2} \frac{1}{4 \pi}\left(\frac{k T_{I C}}{G M m_{H}}\right)^{1 / 2}\left(R_{m i n}^{-1 / 2}-R_{m a s}^{-1 / 2}\right) \\
& =4 \times 10^{61} \mathrm{~cm}^{-3} \frac{L_{38}^{2} f^{2}}{T_{7}^{3 / 2} R_{\min 9}^{-1 / 2}}
\end{aligned}
$$

The results presented so far in this section divide the disk into several qualitatively different zones ordered according to increasing radial distance from the X-ray source: (i) The zone where the coronal temperature is tied to the disk photospheric temperature by Compton cooling; (ii) The optically thick coronal zone, where the vertical column density is limited by radiative transfer effects and where the mean ionization parameter may exceed $\Xi_{h}^{+}$; (iii) The optically thin coronal zone, where the mean ionization parameter is $\Xi_{h}^{+}$and the corona is partially cooled by $X$-rays reprocessed in the photosphere; and (iv) The wind zone where the corona is not bound to the disk. The distinctions between these regions are displayed schematically in Figure 1.

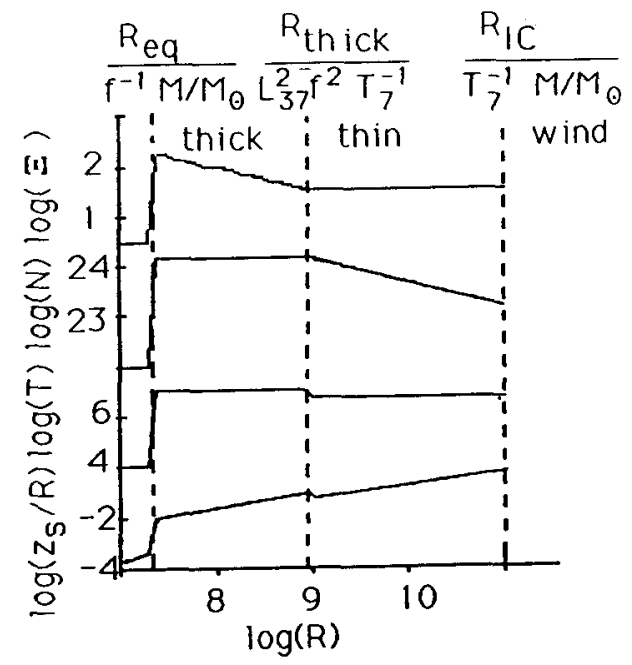

Figure 1.

Inherent in the discussion so far has been the assumption that the flux pro- 
portionality constant, $f$, is known. This factor depends on the detailed geometrical relationship between the compact object and the disk, i.e. the disk flaring angle and the size of the compact object, and on the effects of radiative transfer in the corona. It is possible that the corona may be self-sustaining in the sense that scattered Xrays may be dominant in determining the flux which excites the corona. However, as of this writing there exists no successful calculation of the efficiency of this process. London (1982) showed that in the absence of Compton cooling radiative transfer effects led to a value of $f \simeq 0.01-0.1$ for $X$-ray and disk properties appropriate to low mass X-ray binaries, but was unable to find a self-consistent steady transfer solution when Compton cooling of the corona by the disk was included. In fact, as suggested by Fabian, it is possible that there may exist two distinct states for the corona: a low- $f$ Compton cooled state and a high- $f$ non-cooled state. Given this ambiguity, and given the uncertainties in the disk geometry, we consider the value of $f$ to be an important unknown. As shown in the following section, this quantity can be constrained by observations.

\section{OBSERVATIONS}

The eclipse light curves of low mass X-ray binaries, revealing a significant ( 10\%) residual flux during eclipse, provided much of the original motivation for the study of accretion disk coronae. As shown in Figure 1, the ADC scale height can exceed $z_{s} \simeq R \sim R_{s t a r}$ at large radii, where $R_{s t a}$, is the radius of the companion star. The eclipse flux depends on the Thompson depth of this material, which may be $\sim 0.1$ if $f \sim 0.1$. ADC models have been successfully fitted to the orbital light curves of the high-inclination binaries 4U1822-37 (White and Holt, 1982), 4U2129+47 (BM; White and Holt, 1982; McClintock, et al., 1982), and Cyg X-3 (White and Holt, 1982; Molnar, 1986) observed by proportional counter experiments on the HEAO-1 and Einstein satellites. As suggested by Kahn, higher spectral resolution $(\lambda / \Delta \lambda \geq 20)$ observations of these light curves will test ADC models more severely, since they will allow limits to be set on the existence of photoabsorption edges, and hence on the degree of ionization of the ADC gas.

\subsection{Iron K Emission Lines}

Another observational test of ADC models is provided by the iron $\mathrm{K}$ line emission from low mass X-ray binaries (LMXRB). Such emission has been detected by the gas scintillation proportional counter detectors (GSPC's) on the EXOSAT and Tenma satellites.

A model calculation of the dependence of the emissivities of the $\mathrm{K}$ lines from the various stages of iron on radial and vertical position in an ADC, embodying the ADC structure as summarized in the previous section along with detailed calculations of the atomic physics affecting the line emission, are displayed in figure 2(a) (Kallman and White, 1988). Figure 2(b) displays the emitted line flux in the 6.2 - $7 \mathrm{KeV}$ energy band at radii corresponding to those displayed in Figure 2(a). The 
panels on the left show the spectrum emitted at each radius as it would be viewed by a spectrometer with a resolution $\Delta \varepsilon / \varepsilon=0.002$, roughly that attainable by the best $\mathrm{X}$-ray spectrometers contemplated for the future. The panels on the right show the spectrum as it would be viewed by a spectrometer with a resolution approximately that of a GSPC, $\Delta \varepsilon \sim 100 \mathrm{eV}$. The broadening processes included in the calculations of Figure 2(b) include blending of multiple components from different ionization stages and Compton scattering. The components from various ion ${ }^{-1}$ stages are apparent as the narrow emission spikes in Figure 4a. Comptonization results in the broader "pedestals" under the narrow components. The narrow unscattered line components all contain more energy than do the broadened Compton scattered components, reflecting the fact that the lines are always emitted at Thompson depths $\tau_{T h} \leq 1$. The ratio of the scattered to unscattered components is greatest at the smallest radius, $10^{7} \mathrm{~cm}$. This is consistent with equation (4), showing that the greatest column densities occur at the smallest radii. Broadening due to blending of multiple components results in a line width of approximately $0.5 \mathrm{KeV}$, and so dominates over Compton broadening for the ADC conditions considered here. The line blends are dominated by emission from ion stages Fe XXV and below, and so have centroid energies in the range $6.2-6.7 \mathrm{KeV}$. In the optically thin coronal region, the line blend is receives a strong contribution from the $6.4 \mathrm{KeV}$ line. These photons are emitted by material at low ionization (Fe XVII and below) in the disk photosphere and chromosphere; such emission is weak or absent in the optically thick coronal region owing to attenuation of the $\mathrm{K}$ continuum photons.
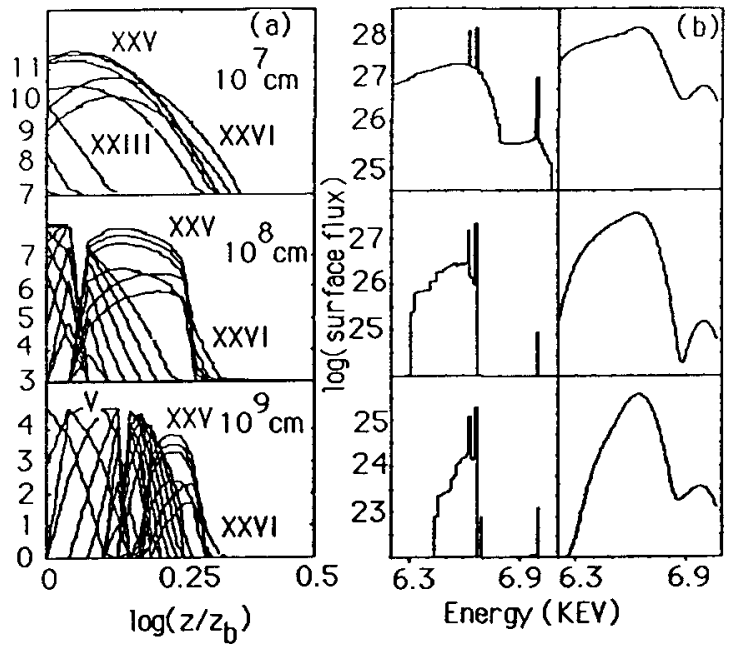

Figure 2

The models shown in Figure 2 may be compared with the results from 
EXOSAT compiled by White, et al. (1986) which show that for most objects the line centroid energy is $\simeq 6.7 \mathrm{KeV}$, and the width (Full Width Half Maximum) is $F W H M \simeq\langle\Delta \varepsilon\rangle \simeq 1 \mathrm{KeV}$. This suggests that the standard ADC model can account for the observed line centroid energies if the flux incident on the disk is relatively high, $f \simeq 0.1$, and if the lines are emitted at small distances from the compact object, $\mathbf{R} \leq 10^{8} \mathrm{~cm}$. If so, the line emission regions must shield the disk at larger radii from X-rays in order to avoid an excess of narrower, lower energy line emission, and the observed width must be due to rotation or some other broadening mechanism. Rotation causes broadening $\Delta \varepsilon / \epsilon=\left(G M / R c^{2}\right)^{1 / 2}=0.24 \mathrm{KeV}(\varepsilon / 6.4 \mathrm{KeV})^{-1} R_{8}^{-1 / 2}$ for Kepler motion around a $1 M_{\odot}$ compact object, so that radii $R \simeq 10^{7} \mathrm{~cm}$ are required to provide the observed broadening.

\subsection{Soft X-ray Lines}

The wavelength range between 10 and $20 \AA$ contains a large number of emission lines which are useful diagnostics of accretion disk coronae. The high spectral density of lines necessitate spectrometers with higher sensitivity and spectral resolution than can be obtained with proportional counters or GSPC's. Line emission in the $0.5-2 \mathrm{KeV}$ energy range due to the $\mathrm{L}$ shell transitions of iron and to the $K$ shell transitions of medium- $Z$ elements such as nitrogen and oxygen from several of the brightest low mass X-ray binaries (Kahn, Seward and Chlebowski, 1982; Vrtilek et al., 1986a, b) have been detected by the transmission grating experiments on the Einstein and EXOSAT observatories.

The interpretation of soft X-ray line spectra is considerably more complicated than that of the Iron K lines. Attempts at model fitting (Vrtilek et al. 1986a,b) have shown that neither models of constant temperature mechanically heated gas (Raymond and Smith, 1977) nor models of photoionized constant density clouds (Kallman and McCray, 1982) give adequate fits to all the line strengths. Furthermore, the atomic processes which affect this line emission are subject to considerable uncertainty. A notable example is dielectronic recombination at temperatures much less than the ionization potential of the most abundant ions, which is likely to be important in photoionization-dominated gases (uncertainties in these rates are discussed in more detail elsewhere in this volume).
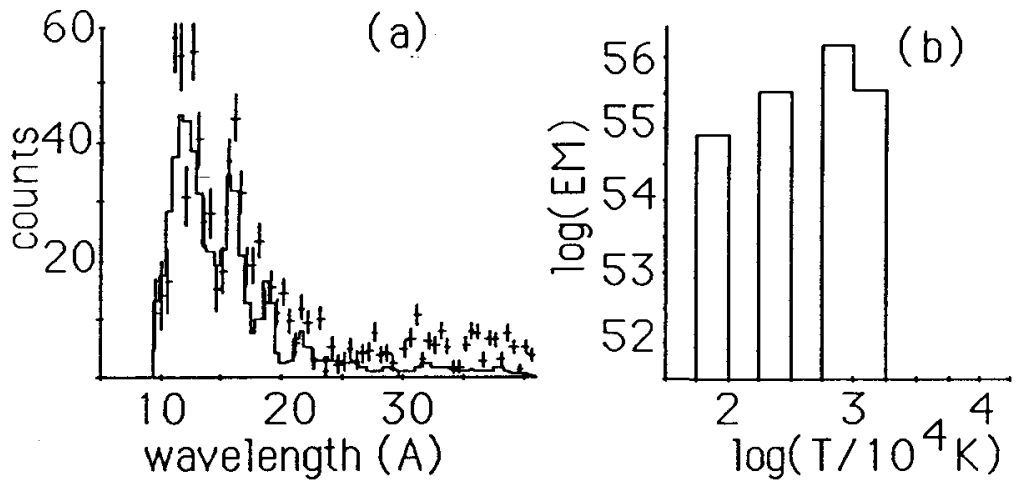

Figure 3 
An attempt at fitting such soft $\mathrm{X}$-ray line spectra to a more complicated model is shown in Figure 3(a). The line spectrum of Cyg X-2 as observed by the objective grating spectrograph (OGS) on the Einstein observatory satellite ("state $G$ " of Vrtilek et al. ,1986b) is displayed (crosses), together with a model consisting of a multi-temperature optically thin mechanically heated gas. The line spectrum is dominated by approximately four emission features, the $12-14 \AA$ features which are likely to be dominated by Fe XVIII - XXII, the $16 \stackrel{\AA}{A}$ feature which may be due to O VIII L $\beta$ or to Fe XVII - XVIII, and the $19 \AA$ feature due to O VIII L $\alpha$. The locations of these strong lines are all matched by the models, and $\chi^{2}$ per degree of freedom is $\mathbf{0 . 7}$ for this model fit shown. In the absence of emission lines the bremsstrahlung continuum alone fits the data with $\chi^{2}$ per degree of freedom of 1.4 , demonstrating that the lines are statistically significant and that the model fit is acceptable. The emission measure distribution, displayed in figure 3(b), ranges from $\log (T)=6.25$ to $\log (T)=7.25$, reflecting the presence in the observed spectrum of emission from ions ranging from Fe XVII to Fe XXIII. Similar results have been obtained from the analysis of the OGS spectra of several other low mass X-ray binaries (Kallman, Vrtilek, and Kahn, 1989).

The emission measures required to fit the Cyg X-2 OGS spectra are much less than those estimated in equation (6), suggesting that only a fraction $\sim 10^{-4} / f$ of the corona will be cool and neutral enough to emit soft X-ray lines. Furthermore, the observed widths of the lines, $\lambda / \Delta \lambda \geq 20$ provide a limit on the rotational broadening and hence on the radius of the emission region, $R \geq 10^{8} \mathrm{~cm}\left(M / M_{\odot}\right)$. Therefore, the soft X-ray emission lines probe a region which is physically distinct from the region responsible for most of the Iron $\mathrm{K}$ line emission discussed in the previous subsection.

\section{SUMMARY}

In spite of the fact that many of the properties of accretion disk coronae can be estimated in a simple way, and that they provide a successful and attractive explanation for the $\mathrm{X}$-ray light curves of many low mass binaries, there remain a number of outstanding problems, including: (i) What is $f$ ? The Sco X-1 Iron $K$ line observations suggest $f \geq 0.1$ in the inner disk regions $\left(R \leq 10^{8} \mathrm{~cm}\right)$ and much smaller values at larger radii, while the calculations of London (1983) suggest $f \simeq 0.01$ at all radii if the Eddington ratio is $\sim 0.1$. Soft $X-r a y$ line emission requires $f \geq 10^{-4}$ for $R \geq 10^{8} \mathrm{~cm}$. The eclipse light curves of high inclination low maxx Xray binaries require $f \geq 0.1$ at the largest disk radii. (ii) Modelling of the Soft X-ray Emission lines: The full power of the soft X-ray emission line observations can't be utilized until uncertainties concerning the atomic physics affecting recombination line emission (and other processes which are more familiar from the study of mechanically heated gases) are resolved. (iii) Modelling of Wind Flow: The gas flow in the disk wind region may be observationally distinguishable from the bound coronal region, for example owing to the presence of material at $T \leq T_{I C}$ far above the disk plane. (iv) Further Observations of Emission Lines: If the Fe $\mathrm{K}$ 
line widths are due to rotation, they are predicted to have a characteristic double peaked structure which will be resolvable with future high resolution spectrometers. The soft X-ray emission lines are known to vary on timescales less than $10^{4} \mathrm{sec}$; observations with sufficient sensitivity to resolve the fastest such variability will provide a lower limit on the size of the region responsible for this emission.

\section{REFERENCES}

Begelman, M., McKee, C. E., and Shields, G. B., 1983, Ap. J., 271, 70.

Begelman, M. and McKee, C. E., 1983, Ap. J., 271, 89 (BMS).

Fabian, A., Guilbert, P., and Ross, R.R., 1982, MNRAS, 199, 1045.

Field, G. B., 1965, Ap. J., 142, 531.

Kahn, S.M., Seward, F.D., and Chlebowski, T., 1984, Ap. J., 283, 286.

Kallman, T. R., 1984, Ap. J., 280, 269.

Kallman, T. R., and McCray, R. A., 1982, Ap. J. Suppl., 50, 263.

Kallman, T. R. and Mushotzky, R., 1985, Ap. J., 292, 49.

Krolik, J.H., and Kallman, T.R., 1984, Ap. J., 286, 366.

Krolik, J.H., and Kallman, T.R., 1987, Ap. J. Lett., 320, L5.

Krolik, J. H., McKee, C. F., and Tarter, C. B., 1981, Ap. J., 249, 422.

London, R., 1982, in Cataclysmic Variables and Low Mass $X$-ray Binaries, J. Patterson and D. Lamb, eds..

McClintock, J., London. R.A., Bond. H.E., and Grauer, A.D., 1982, Ap. J., 258, 245 .

Molnar, L., 1986, in The Physics of Accretion onto Compact Objects, K.O. Mason, M. Watson, and N.E. White, eds. (Berlin: Springer).

Raymond J.C., and Smith, B. H., 1977, Ap. J. Supp., 35, 419.

Shakura, N. I., and Sunyaev, R.A., 1971, Astron. and Astrophys., 24, 337.

Shields, G.A., McKee, C.F., Lin, D.C., and Begelman, M. 1986, Ap. J., 306, 90.

Vrtilek, S.D., Kahn, S.M., Grindlay, J.E., Helfand, D.J., and Seward, F.E., 1985, Ap.J., 307, 698 .

Vrtilek, S.D., Helfand, D.J., Halpern, J.P., Kahn, S.M., and Seward, F.D., 1986, Ap. J., .

Vrtilek, S. D., Swank, J., and Kallman, T., 1988, Astrophys. J., 326, 186.

White, N.E., and Holt, S.S., 1982, Ap. J., 257, 318.

White, N.E. et al., 1986, M.N.R.A.S., 218, 129. 